\title{
Sensor Fault Diagnosis of Locomotive Electro-Pneumatic Brake Using an Adaptive Unscented Kalman Filter
}

\author{
Dianzhu Gao,, ${ }^{1,2}$ Jun Peng, ${ }^{2}$ Yunyou Lu, ${ }^{1}$ Rui Zhang, ${ }^{1}$ Yingze Yang $\triangle{ }^{2}$ and Zhiwu Huang ${ }^{1}$ \\ ${ }^{1}$ School of Automation, Central South University, Changsha 410075, China \\ ${ }^{2}$ School of Computer Science and Engineering, Central South University, Changsha 410075, China
}

Correspondence should be addressed to Yingze Yang; yangyingze@csu.edu.cn

Received 15 July 2021; Revised 30 September 2021; Accepted 27 November 2021; Published 16 December 2021

Academic Editor: Hongjin Wang

Copyright ( 2021 Dianzhu Gao et al. This is an open access article distributed under the Creative Commons Attribution License, which permits unrestricted use, distribution, and reproduction in any medium, provided the original work is properly cited.

\begin{abstract}
Normal operation of the pressure sensor is important for the safe operation of the locomotive electro-pneumatic brake system. Sensor fault diagnosis technology facilitates detection of sensor health. However, the strong nonlinearity and variable process noise of the brake system make the sensor fault diagnosis become challenging. In this paper, an adaptive unscented Kalman filter- (UKF-) based fault diagnosis strategy is proposed, aimed at detecting bias faults and drift faults of the equalizing reservoir pressure sensor in the brake system. Firstly, an adaptive UKF based on the Sage-Husa method is applied to accurately estimate the pressure transients in the equalizing reservoir of the brake system. Then, the residual is generated between the estimated pressure by the UKF and the measured pressure by the sensor. Afterwards, the Sequential Probability Ratio Test is used to evaluate the residual so that the incipient and gradual sensor faults can be diagnosed. An experimental prototype platform for diagnosis of the equalizing reservoir pressure control system is constructed to validate the proposed method.
\end{abstract}

\section{Introduction}

The electro-pneumatic brake system has shown the extensive applications in passenger trains, metros, and heavy haul trains because of its fast response time and high reliability [1]. Locomotive electro-pneumatic brake is a crucial component which has an important function for the operational safety of the train. Faults in braking systems can lead to a reduction in locomotive braking performance and even induce safety accidents. Therefore, early detection and isolation of faults in the braking system are necessary [2]. Pressure sensors are vital components in the brake system because their reliability and measuring accuracy are crucial to achieving the accurate pressure control and approving braking performance.

The fault diagnosis of the equalizing reservoir pressure sensor is a challenging task. The brake system is composed of the electric, pneumatic, and mechanical subsystem, showing a sophisticated nonlinearity [1]. The energy transmitting medium of the braking force is compressed air, and the compressibility of air makes the system highly nonlinear [3], which makes it difficult to build a precise mathematical model of the brake system. Furthermore, the process noise and measurement noise in the braking process, which are caused by the harsh and noisy working environment, make the fault diagnosis of the brake become more challenging.

Recent years, many studies have developed sensor fault diagnosis methods [4-8]. There are three main categories of sensor fault diagnosis methods: the redundancy method and the knowledge-based method and the model-based approaches. The redundancy method is implemented by the comparison of measurements among several sensors, which has been used in wireless sensor networks [9] or the aerospace system [10], such as satellite attitude control systems [11]. The minimum degree of sensor redundancy necessary to pinpoint the distinction between sensor faults and system faults in the monitoring process is determined in [12]. However, the redundancy methods require additional 
hardware sensors, showing less cost-effectiveness, which are not appropriate for the locomotive electro-pneumatic brake system [13].

The development of computer technology has provided a new method for fault diagnosis technology. Knowledgebased method uses an expert system to locate and diagnose sensor faults and does not require a quantitative mathematical model. A fuzzy expert system is established to locate sensor faults [14], and the residual generation and residual evaluation are analyzed in [15], showing its instantaneous handling capability for the fault. The problem of sensor fault recognition is considered pattern recognition in [2]. The sample data is acquired and trained to obtain a classifier, and then, the data is matched according to the classification rules. However, it is general that knowledge-based methods require a large enough amount of data, which means that many types and numbers of sensors need to be added in the brake system.

For model-based methods, the Kalman filter and its enhanced varieties are widely utilized $[5,7,16]$ because of their robustness to process and measurement noise and their efficient real-time performance [17]. However, the Kalman filter is not available for the brake system because of its intrinsic nonlinear properties. Therefore, an unscented Kalman filter (UKF) is proposed to address the nonlinear problem. The UKF, which applies the unscented transform to calculate the mean and variance of measurement and process noise, has higher accuracy than the extended Kalman filter $[18,19]$. However, the process noise and covariance matrices of measurement for UKF are generally assumed to be stable. And it is difficult to determine the covariance matrices in practical applications. The fault diagnosis method will suffer from performance degradation if the model uncertainty is not well defined by the process noise covariance [20]. To overcome the difficulty, the adaptivity of UKF should be improved. That is, the covariance matrices of measurement and process noise should be adaptively adjusted $[8,21,22]$.

This paper proposes an adaptive UKF-based scheme to detect bias faults and drift faults of the equalizing reservoir pressure sensor. For the locomotive electro-pneumatic brake system, different from existing UKF-based fault diagnosis methods, the proposed scheme can detect incipient and gradual sensor faults. The scheme introduced the SageHusa mechanism to accurately estimate the pressure transients in the equalizing reservoir by filtering out the measurement noise and the changing process noise of the brake system. Further, the Sequential Probability Ratio Test is utilized to evaluate the residual, the difference between the estimated pressure, and the online sensor measurement. By combining the Sage-Husa mechanism and Sequential Probability Ratio Test, the proposed scheme can detect the incipient and gradual sensor faults of the locomotive electro-pneumatic brake system. The main contributions in this paper include the following:

(i) The mechanism of the electro-pneumatic brake system is analysed adequately, and the accurate analytical pressure model is established (ii) The adaptive UKF is applied to estimate the system output pressure, improving the robustness of the fault diagnosis approach under the uncertainty and noise

(iii) The Sequential Probability Ratio Test is introduced to evaluate the residual to minimize the occurrence of misinformation or false detection in fault diagnosis

The rest of this paper is organized as follows. Section 2 gives a description of the brake system and builds the mathematical model. Section 3 introduces the theory of adaptive $\mathrm{UKF}$ and presents the fault diagnosis scheme of the pressure sensor bias and drift faults. Section 4 shows the experimental results and analysis. Finally, the conclusion is drawn in Section 5 .

\section{System Model and Problem Formulation}

2.1. Principle of the Electro-Pneumatic Brake System. The electro-pneumatic brake system (see Figure 1) consists of the mechanical, pneumatic, and electric subsystem. The mechanical subsystem is the foundation brake rigging which mainly consists of the brake pads, drum, and shoes. The pneumatic subsystem consists of many components, including the main reservoir, the brake pipe and chamber, an equalizing reservoir, a relay valve, and a compressor. The electrical subsystem mainly contains a brake control unit (BCU), pressure sensors, and solenoid valves (brake valve and release valve).

2.2. Model of the Equalizing Reservoir Pressure Control System. The ideal gas law equation is as follows:

$$
P=\frac{n R T}{V}
$$

which describes the quantitative relation among pressure, air temperature, and volume of a chamber, where $n, R, P, T$, and $V$ represent the number of moles of the gas, the gas constant, the absolute pressure, the absolute air temperature, and the chamber volume, respectively. Assuming the volume $V$ is invariable, taking the derivative of the equation with respect to time, we can get

$$
\dot{P}=\frac{R T}{V} q_{m}
$$

where $q_{m}$ is the mass flow in the chamber. According to Bernoulli's equation for adiabatic and isentropic airflow, $q_{m}$ is calculated as follows [23]:

$$
q_{m}=f\left(P_{\mathrm{u}}, P_{\mathrm{d}}\right)=\left\{\begin{array}{l}
* 20 c \frac{P_{\mathrm{u}} C_{1} A}{\sqrt{R T}} \sqrt{\gamma\left(\frac{2}{\gamma+1}\right)^{(\gamma+1) /(\gamma-1)}}, \frac{P_{\mathrm{d}}}{P_{\mathrm{u}}} \leq 0.528, \\
\frac{P_{\mathrm{u}} C_{2} A}{\sqrt{R T}} \sqrt{\frac{2 \gamma}{\gamma-1}} \sqrt{\left(\frac{P_{\mathrm{d}}}{P_{\mathrm{u}}}\right)^{2 / \gamma}-\left(\frac{P_{\mathrm{d}}}{P_{\mathrm{u}}}\right)^{(\gamma+1) / \gamma}}, 0.528<\frac{P_{\mathrm{d}}}{P_{\mathrm{u}}} \leq 1,
\end{array}\right.
$$




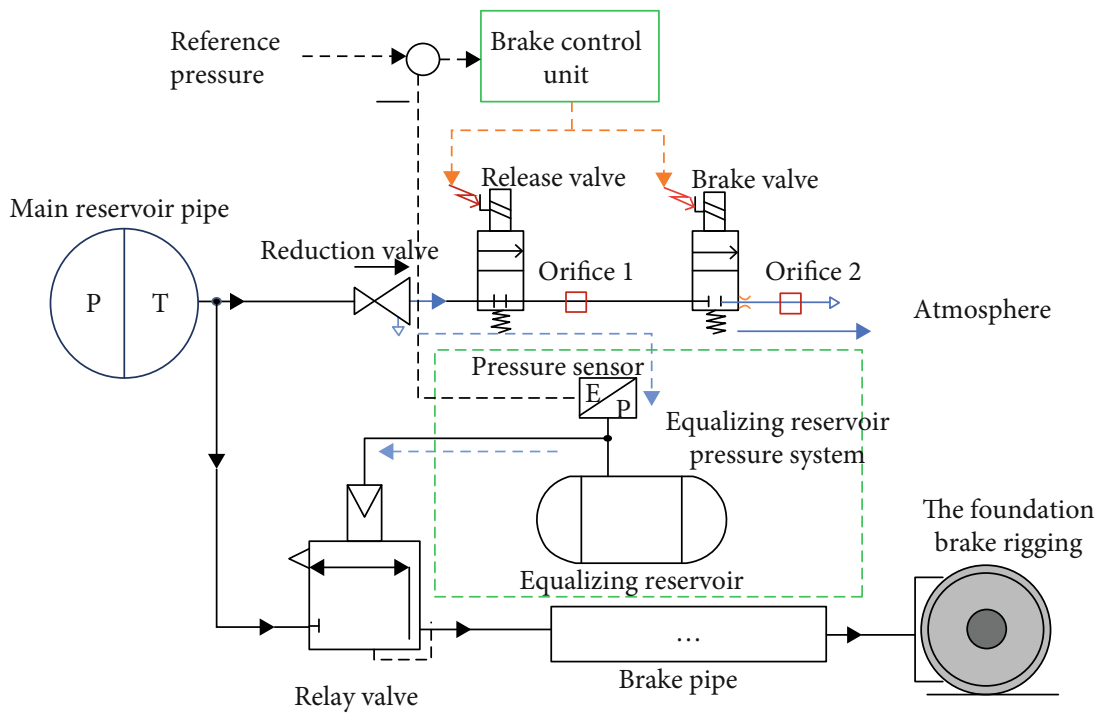

Figure 1: The schematic of the locomotive electro-pneumatic brake system.

where $R$ and $T$ have the same meanings as those in the ideal gas law equation. $P_{\mathrm{d}}$ and $P_{\mathrm{u}}$ are the downstream pressure and upstream pressure, respectively. $C_{1}$ and $C_{2}$ are the flow rate coefficients. $\gamma$ represents the adiabatic exponent of air, and $A$ represents the orifice passage area. By combining (1) and (2), the equalizing reservoir pressure transients of the brake system in different operating modes can be formulated as (4), (5), and (6).

The equalizing reservoir pressure dynamics in the release process is formulated as

$$
\dot{P}=\left\{\begin{array}{l}
\frac{P_{s} C_{1} A_{1} \sqrt{T R}}{V} \sqrt{\gamma\left(\frac{2}{\gamma+1}\right)^{(\gamma+1) /(\gamma-1)}}, \frac{P}{P_{s}} \leq 0.528, \\
\frac{P_{s} C_{2} A_{1} \sqrt{T R}}{V} \sqrt{\frac{2 \gamma}{\gamma-1}} \sqrt{\left(\frac{P}{P_{s}}\right)^{2 / \gamma}-\left(\frac{P}{P_{s}}\right)^{(\gamma+1) / \gamma}}, 0.528<\frac{P}{P_{s}} \leq 1,
\end{array}\right.
$$

where $P$ is the equalizing reservoir pressure, $P_{s}$ is the main reservoir pressure, $A_{1}$ represents the orifice passage area of the release valve, and $V$ is the equalizing reservoir volume.

The pressure in the equalizing reservoir remains steady in the hold mode. Then, the pressure dynamics can be described as

$$
\dot{P}=0 \text {. }
$$

The equalizing reservoir pressure dynamics in the braking process is formulated as

$$
\dot{P}=\left\{\begin{array}{l}
-\frac{P C_{3} A_{2} \sqrt{T R}}{V} \sqrt{\gamma\left(\frac{2}{\gamma+1}\right)^{(\gamma+1) /(\gamma-1)}}, \frac{P_{o}}{P} \leq 0.528, \\
-\frac{P C_{4} A_{2} \sqrt{T R}}{V} \sqrt{\frac{2 \gamma}{\gamma-1}} \sqrt{\left(\frac{P_{o}}{P}\right)^{2 / \gamma}-\left(\frac{P_{o}}{P}\right)^{(\gamma+1) / \gamma}}, 0.528<\frac{P_{o}}{P} \leq, 1
\end{array}\right.
$$

where $P_{o}$ is the atmosphere pressure, $A_{2}$ represents the orifice passage area of the brake valve, and $C_{3}$ and $C_{4}$ are the flow rate coefficients.
From (4) and (6), we can know that the brake system is strongly nonlinear; thus, we choose the UKF as the pressure estimator. The mathematical model should be as accurate as possible in order to realize an effective fault diagnosis scheme. Therefore, the parameters in the models should be obtained accurately. The equalizing reservoir volume, the orifice passage areas of the release valve, and the brake valve can be measured directly. However, the flow rate coefficients in (4) and (6) need to be identified. In this paper, the flow rate coefficients $C_{1} \sim C_{4}$ are identified by the least square method [24]. The validity of the model is tested by experiments, which is described in Section 4.

\section{The Proposed Sensor Fault Diagnosis Method}

The theory of adaptive UKF and the proposed sensor fault diagnosis method are introduced in this section. Firstly, the principle of adaptive UKF is developed, and then, the algorithm is applied to fault diagnosis of the equalizing reservoir pressure sensor.

3.1. The Theory of Adaptive UKF. Based on the theory of traditional UKF, the prior statistics of the process noise is used to compensate for the changing model uncertainty [25], which is adaptively corrected by the Sage-Husa noise estimator. The UKF is used for the discrete system generally. The general form of a discrete nonlinear system is defined by

$$
\left\{\begin{array}{l}
x_{k+1}=f\left(x_{k}, y_{k}\right)+q_{k}=F_{k} x_{k}+B_{k} u_{k}+q_{k}, \\
y_{k}=h\left(x_{k}\right)+r_{k},
\end{array}\right.
$$

where $u_{k}$ and $x_{k}$ are the input vector and $n$-dimensional state vector, respectively. $y_{k}$ is the $m$-dimensional observation vector. $q_{k}$ and $r_{k}$ represent the process noise and measurement noise, respectively, which are the Gaussian white noise with zero mean. 
Normally, the statistics of the measurement noise and process noise is unvarying in the UKF. However, the process noise of the brake system is varying and difficult to be determined. The measurement noise lies on the accuracy of the pressure sensor and is relatively constant. The statistics of process noise is described by the covariance matrices $Q$. Similarly, the measurement noise $R$ can be calculated from historical measurements. Then, the SageHusa method is applied to tune the covariance matrices $Q$ adaptively. The Sage-Husa suboptimal noise estimator is depicted as follows [26]:

$$
\left\{\begin{array}{l}
d_{k}=\frac{(1-b)}{\left(1-b^{k}\right)}, \\
v_{k}=y_{k}-h\left(\bar{x}_{k \mid k-1}\right), \\
Q_{k}=\left(1-d_{k-1}\right) Q_{k-1}+d_{k-1}\left[K_{v} v_{k} v_{k}^{T} K_{k}^{T}+P_{k}-\sum_{i=0}^{2 n} \omega_{i}^{c}\left(\chi_{i, k \mid k-1}-\bar{x}_{k \mid k-1}\right)\left(\rho_{i, k \mid k-1}-\bar{y}_{k \mid k-1}\right)^{T}\right],
\end{array}\right.
$$

where $b \in(0.95,0.99)$ is the forgetting factor and $K_{k}$ is the Kalman gain.

The more the process and measurement noise change, the higher the value of $b$. The Sage-Husa noise estimator cannot normally work when the prior statistical characteristic of noises is unknown; otherwise, the filter will diverge [27]. The statistics of the measurement noises in the brake system can be obtained according to historical measurements.

\subsection{Residual Evaluation through the Sequential Probability} Ratio Test. In order to minimize the occurrence of misinformation or false detection in fault diagnosis, [28] proposed an improved Sequential Probability Ratio Test (SPRT) method. In this method, statistical hypothesis tests are used, where $H_{0}$ and $H_{1}$ are supposed to be the nonfault hypothesis and faulty hypothesis, respectively [29]. The residuals under fault-free condition conform to a normal random variable (variance value $\sigma$ and mean value $\mu_{0}$ ), while the residuals in faulty condition have the same variance value $\sigma$, whose mean value is $\mu_{1}$. The log-likelihood ratio is calculated as follows:

$$
L(k)=\ln \frac{p\left(r_{i} \mid H_{0}\right)}{\left(r_{i} \mid H_{1}\right)}=\frac{k\left(\bar{r}_{k}-\mu_{0}\right)^{2}}{2 \sigma^{2}}, \quad \bar{r}_{k}=\frac{1}{k} \sum_{i=1}^{k} r_{i} .
$$

The fault detection is then converted into detecting the changes of the residual mean. When there is no fault, $L(k)$ is near to zero. When there is a fault, $\bar{r}_{k}$ would be away from $\mu_{0}$ and $L(k)$ would be away from zero.

A fault is detected when $L(k) \geq T\left(H_{1}\right)$, where $T\left(H_{1}\right)$ is the threshold, $P_{\mathrm{M}}$ is the missing report rate, and $P_{\mathrm{F}}$ is the false alarm rate. In this paper, we set $P_{\mathrm{M}}=0.01, P_{\mathrm{F}}=0.01$, and $T\left(H_{1}\right)=4.595$. When $k$ is too large, $L(k)$ will exceed $T\left(H_{1}\right)$ even though a small deviation between $\bar{r}_{k}$ and $\mu_{0}$ exists. In order to solve the issue, we set an upper bound, 2000 on $k$, and the upper bound is calibrated through experiments.
3.3. The Proposed Sensor Fault Diagnosis Method. The schematic of the proposed sensor fault diagnosis approach is described in Figure 2. It is assumed that the process and measurement noise of the equalizing reservoir pressure system is the Gaussian white noise. Thus, an adaptive UKF can be employed to estimate output pressure according to the inputs and outputs of equalizing reservoir pressure system. The inputs of the equalizing reservoir pressure system are generated by the brake control unit. The outputs of the equalizing reservoir pressure system are measured by a pressure sensor. Then, the residual is generated by subtracting the pressure sensor measurement from the adaptive UKF pressure estimation. Afterwards, the residual is passed through the Sequential Probability Ratio Test to increase the sensing sensitivity. By comparing the preset threshold, the fault detection result can be obtained.

The adaptive UKF is applied as the state estimator in this paper. Based on the conventional UKF algorithm, the measurement noise and process noise covariance is adaptively tuned according to (7) by using the Sage-Husa method. The fault diagnosis scheme is based on the equalizing reservoir pressure system models (3), (4), and (5). Since the system is nonlinear, we choose the Runge-Kutta methods to discretize the system models. Because the UKF algorithm can achieve third-order accuracy of the covariance and posterior mean [18], we use the second-order Runge-Kutta method [30], whose local truncation error is $O\left(h^{3}\right)$ and $h$ is the step size. The process of the proposed sensor fault diagnosis scheme is depicted in Algorithm 1, where the equalizing reservoir pressure, $P$, is chosen as the state variable, and the system state equations (3), (4), and (5) are simplified as $\dot{x}=f(x)$. The observation equation is $y=x$, where $y$ is the pressure sensor measurement. $T=0.02$ (second) is the step size.

\section{Simulation Results and Discussions}

We construct an experimental platform for the equalizing reservoir pressure system and verify the effectiveness and feasibility of the proposed sensor fault diagnosis strategy. The experimental platform (see Figure 3) is part of the real locomotive electro-pneumatic brake.

The detailed parameters of the mathematical models are shown in Table 1, and the flow rate coefficients of the system are identified by the least square method. Firstly, the validity of the mathematical model of the equalizing reservoir pressure system is verified by experiments. Then, bias faults and drift faults are injected into the equalizing reservoir pressure sensor, and fault diagnosis performance of the proposed method is evaluated. Finally, fault diagnosis performance of the proposed method is compared with that of the Luenberger observer.

4.1. Model Verification. Figure 4 depicts the equalizing reservoir pressure transients, where the red line represents the equalizing reservoir pressure measured by a normal sensor and the blue line represents the pressure calculated from the mathematical model. The relative error between sensor measurement and model output is described by the green 


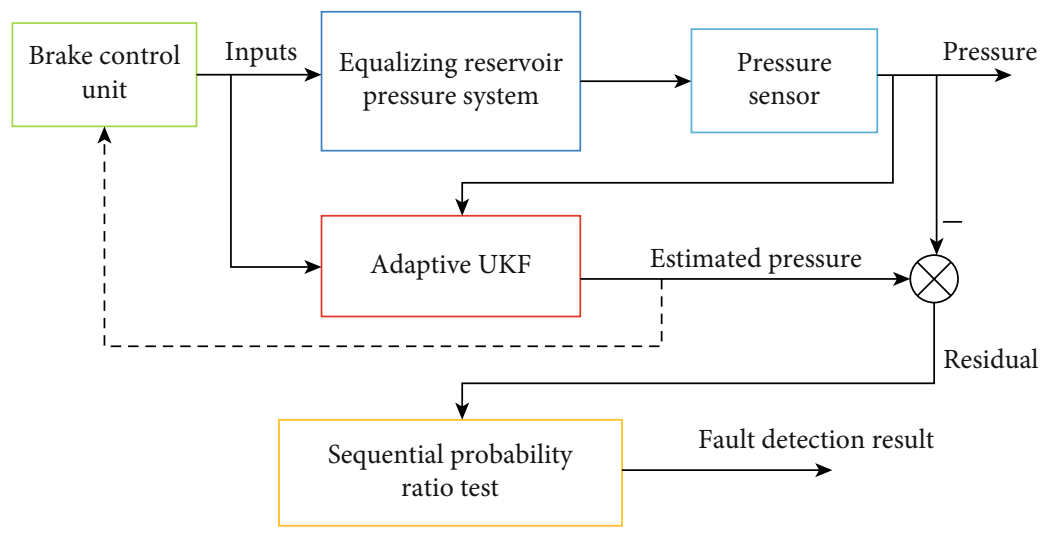

FIGURE 2: Schematic of the proposed sensor fault diagnosis method.

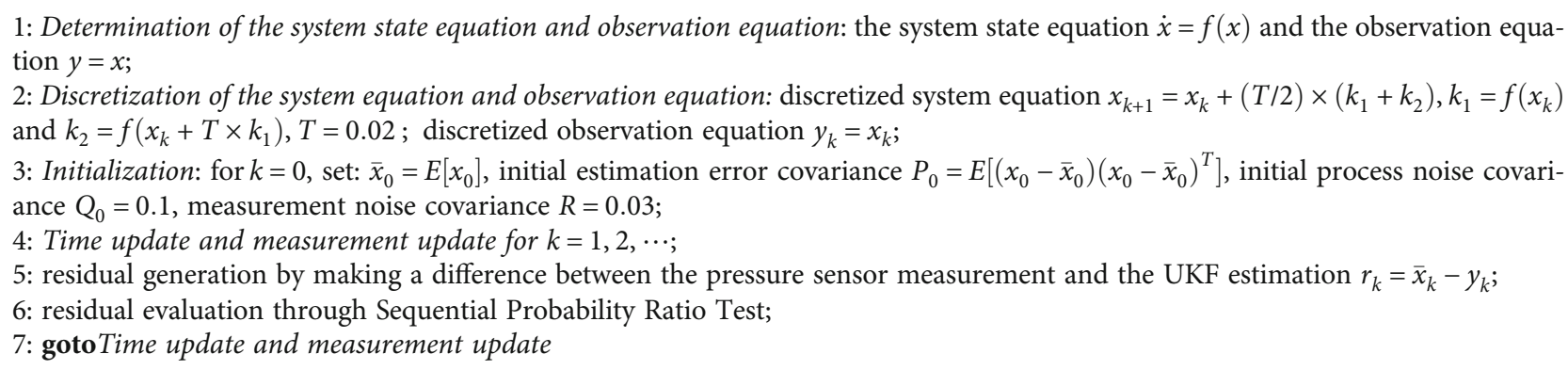

Algorithm 1: The procedure of sensor fault diagnosis.

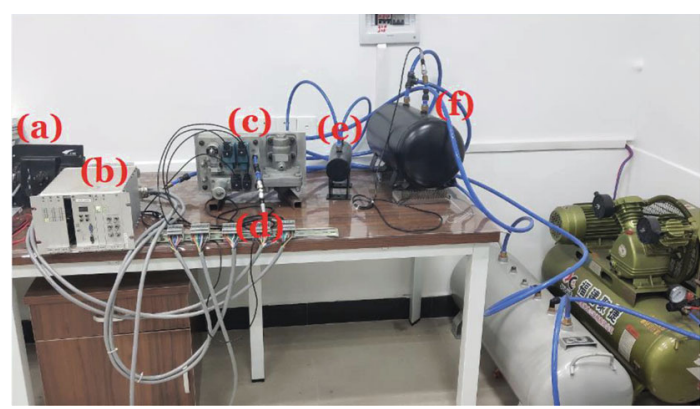

Figure 3: The experimental platform for the equalizing reservoir pressure control system, which is part of the real locomotive electro-pneumatic brake, including the following: (a) upper computer, (b) brake control unit, (c) value and installation gas circuit plate, (d) pressure sensor, (e) equalizing cylinder, and (f) cylinder to simulate a train pipe.

TABle 1: Parameters of the system model.

\begin{tabular}{lccc}
\hline Parameter & Value & Parameter & Value \\
\hline$P_{s}$ & $650 \mathrm{kPa}$ & $P_{o}$ & $101.33 \mathrm{kPa}$ \\
$A_{1}$ & $4 \mathrm{~mm}^{2}$ & $A_{2}$ & $3 \mathrm{~mm}^{2}$ \\
$T$ & $293 \mathrm{~K}$ & $R$ & $287 \mathrm{~J} /(\mathrm{kg} \cdot \mathrm{K})$ \\
$\gamma$ & 1.403 & $V$ & $1.5 \mathrm{~L}$ \\
$C_{1}$ & 0.4593 & $C_{2}$ & 0.4362 \\
$C_{3}$ & 0.2505 & $C_{4}$ & 0.1905 \\
\hline
\end{tabular}

line. The equalizing reservoir pressure transients in the braking process are illustrated (see Figure 5), and the relative error between sensor measurement and model output is plotted by the green line. It can be found that the accuracy of the system model is high adequately.

4.2. Bias Fault Detection. The residuals resulted from sensor bias faults in the release process are shown (see Figure 6), and the bias faults are injected to the sensor at the third second after the release operation. In this figure, the blue line describes the residuals resulting from the bias fault whose magnitude is $1 \mathrm{kPa}$, and the residuals resulting from the bias fault of $2 \mathrm{kPa}$ magnitude are depicted by the red line. We see that the amplitude of the residuals changes after the bias fault occurs (see Figure 6). The larger the fault magnitude, the larger the residual magnitude. The fault detection result of sensor bias faults with different magnitudes is shown in the release process (see Figure 7). In this figure, we can see that the bias fault whose magnitude is $2 \mathrm{kPa}$ is detected, while the bias fault of $1 \mathrm{kPa}$ magnitude has not been detected. This is because the model is not accurate enough. To improve the sensitivity of fault detection, the model needs to be sufficiently accurate.

The residuals of sensor bias faults in the braking process are depicted (see Figure 8), and sensor bias faults with different magnitudes occur at the eighth second after the braking operation. The blue line represents the residuals of the bias fault whose magnitude is $1 \mathrm{kPa}$, and the bias fault of $2 \mathrm{kPa}$ magnitude is represented by the red line. We can know that 


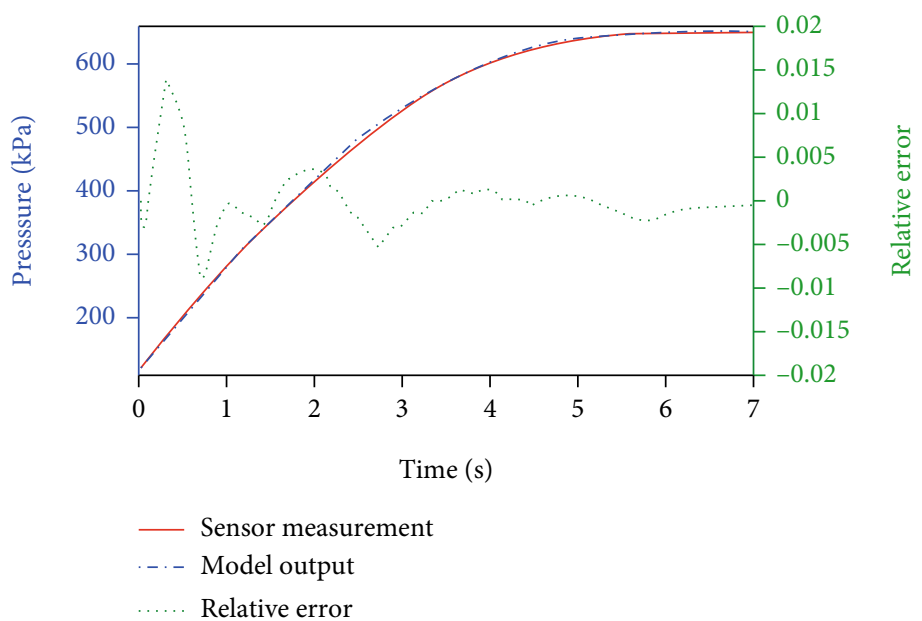

FIGURE 4: Pressure transients measured by a sensor and calculated by a mathematical model in the release process.

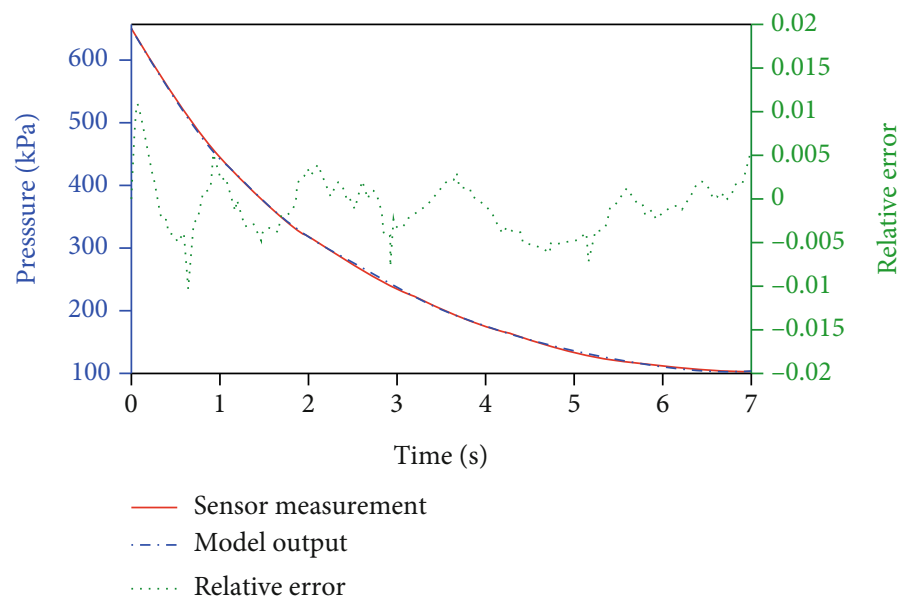

FIGURE 5: Pressure transients measured by a sensor and calculated by a mathematical model in the braking process.

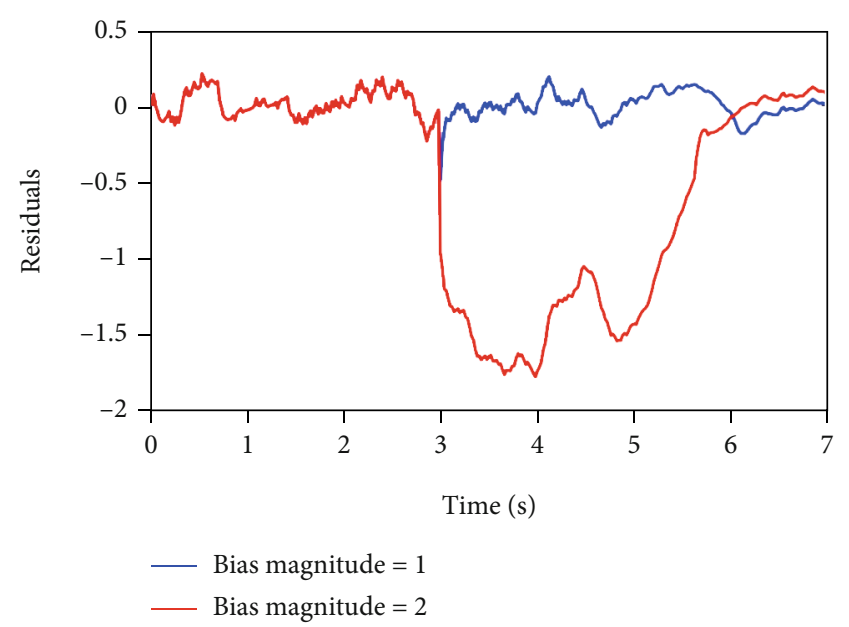

FIGURE 6: Residuals resulted from sensor bias faults with different magnitudes in the release process.

the residual magnitudes change after the bias fault occurs (see Figure 8). The fault detection result of sensor bias faults with different magnitudes is shown in the braking process (see Figure 9). From the figure, we can see that the bias fault of $2 \mathrm{kPa}$ magnitude has been detected, while the bias fault whose magnitude is $1 \mathrm{kPa}$ has not been detected.

4.3. Drift Fault Detection. In this part, the drift fault detection is implemented in the braking and release processes, which is simulated by injecting a varying error to the measurement process, and the error magnitude increases each sampling period.

The residuals resulting from a sensor drift fault in the release process are described (see Figure 10), and the sensor drift fault occurs at the third second after the release operation. The measurement error increases artificially by $0.02 \mathrm{kPa}$ each sampling period to simulate the drift fault. It can be seen that the residual changes slightly after the drift fault occurs (see Figure 10). The sensor drift fault detection results are shown in the release process (see Figure 11), where the log-likelihood ratio, $L(k)$, changes after the fault occurrence. The drift fault is detected about 2.5 seconds after its occurrence. Then, we can conclude that the Sequential Probability Ratio Test method has excellent performance in detecting the gradual fault. The drift fault detection result 


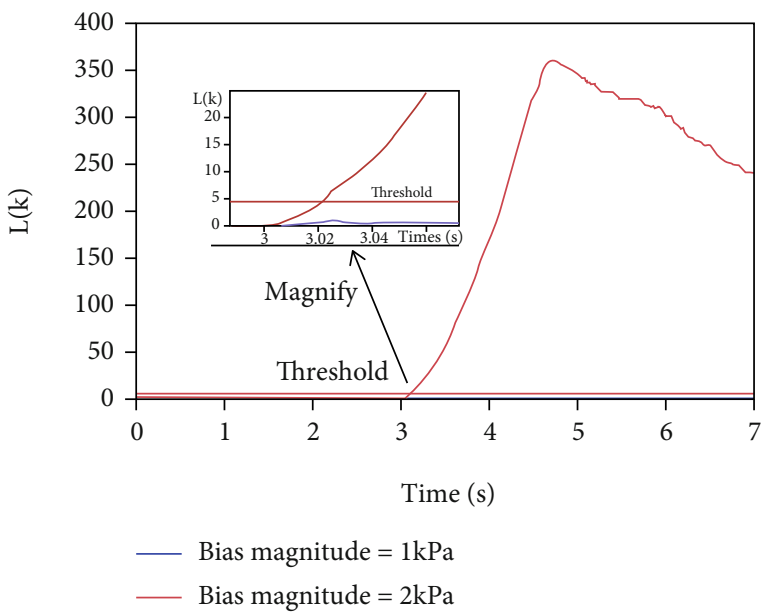

FIGURE 7: Fault detection result of sensor bias faults with different magnitudes in the release process.

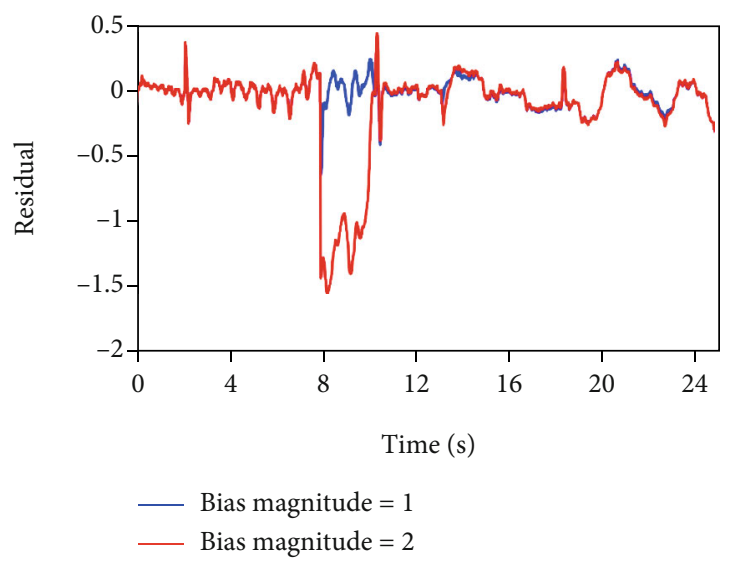

Figure 8: The influence of different magnitudes on residuals resulted from sensor bias faults.

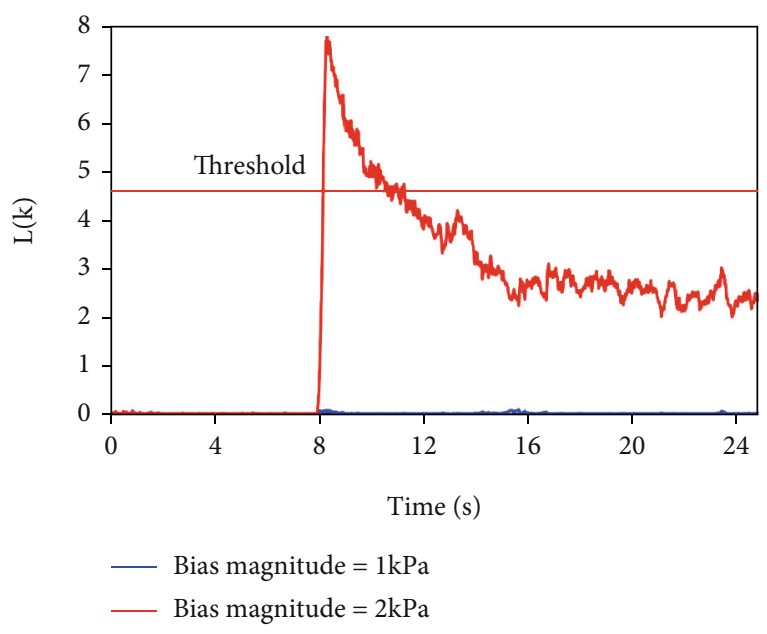

Figure 9: The influence of different magnitudes on sensor bias fault detection result.

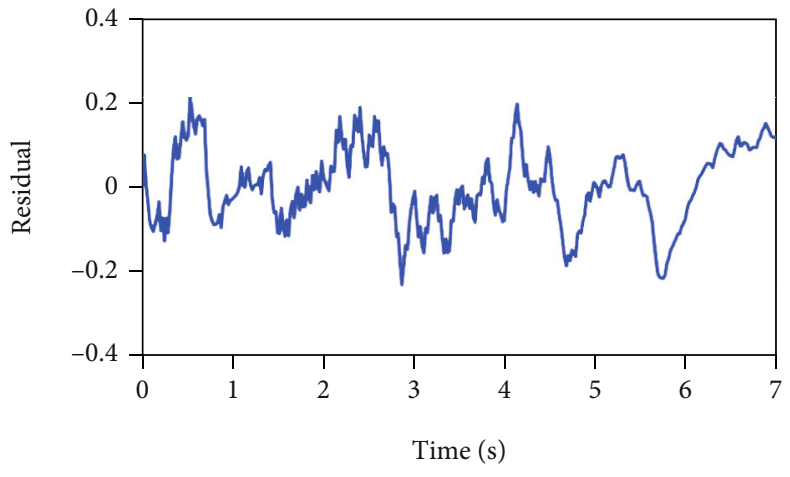

FIGURE 10: Residuals resulted from sensor drift fault in the release process.

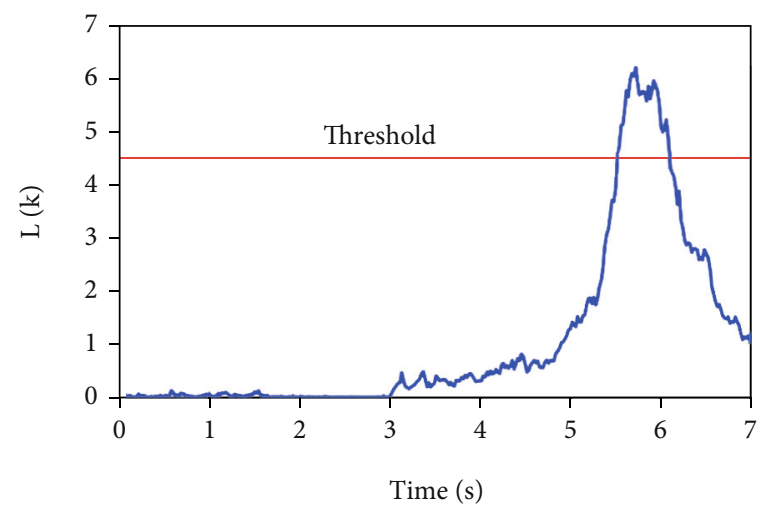

Figure 11: Drift fault detection results in the release process.

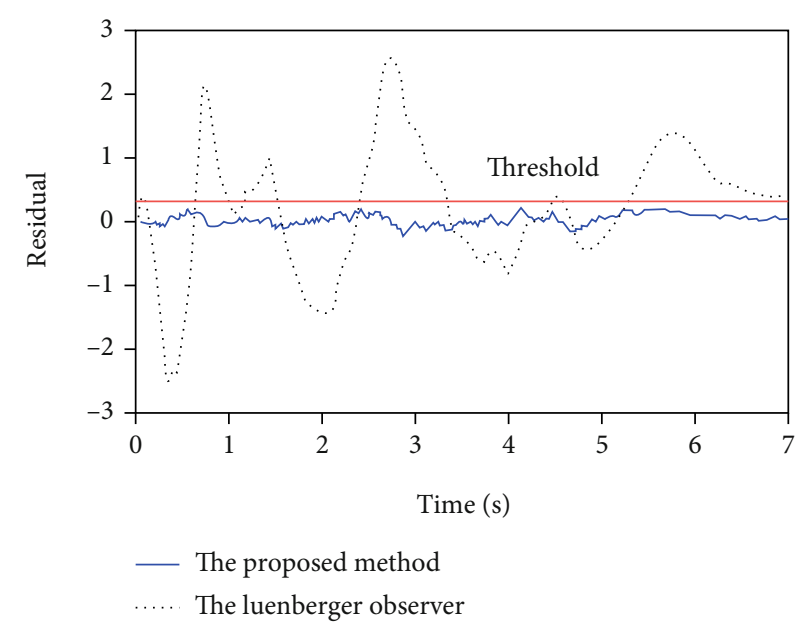

FIgURE 12: The comparison of residuals generated by the proposed methods and Luenberger observer.

of the equalizing reservoir pressure sensor in the braking process is similar to that in the release process.

4.4. Performance Comparison of Sensor Fault Diagnosis Methods. In order to compare the performance of different fault diagnosis methods, the residual of the proposed fault diagnosis method is compared with that of the Luenberger observer during the brake release procedure. Residual comparison of different methods is carried out when the 
locomotive electro-pneumatic brake system is operated under normal conditions. In the experiment, the residuals are generated by subtracting the sensor measurement from the equalization cylinder pressure estimated by the observer and the proposed method based on the adaptive unscented Kalman filter, respectively.

In Figure 12, the blue line describes the residuals of the fault detection method based on the adaptive untraceless Kalman filter. The black line represents the residuals of the Luenberger observer. And the red line represents the fault detection threshold. From Figure 12, it can be seen that there are many false error detections when using the Luenberger observer for fault detection, because the error of the mechanism model is too large. If the threshold is increased to reduce the false positive rate of the Luenberger observer method, the sensitivity of the Luenberger observer method will be reduced. On the contrary, the residual error of the proposed method is much smaller than that of the Luenberger observer method and fluctuates little. By comparing the residuals generated by the two methods and analysing the fault detection results, the conclusion can be drawn that the proposed fault detection method has better accuracy and sensitivity than the Luenberger observer method. This is because the adaptive unscented Kalman filter can filter out the changing process noise and measurement noise and accurately estimate the pressure of the equalizing air cylinder.

\section{Conclusions}

This paper proposes an efficient and novel model-based sensor fault diagnosis algorithm based on UKF for the locomotive electro-pneumatic brake system. For this purpose, the accurate pressure mathematical model is first built. Then, an adaptive UKF is applied to estimate the pressure transients of the equalizing reservoir to improve the algorithm's robustness. The residuals are calculated, and the residual evaluation is implemented by an improved Sequential Probability Ratio Test method. The proposed algorithm can efficiently detect drift faults and bias faults of the equalizing reservoir pressure sensor. Experiments validate the feasibility and effectiveness. The future work that needs to be investigated is to improve the fault detection sensitivity for minor and gradual fault.

\section{Data Availability}

The data used to support the findings of the manuscript are available within the article.

\section{Conflicts of Interest}

The authors declare that they have no conflicts of interest.

\section{Acknowledgments}

This work was supported in part by one grant from the National Natural Science Foundation of China (No.
61803394) and one general project of Hunan Natural Science Foundation (No. 2021JJ30876).

\section{References}

[1] C. Siva Chaitanya, S. C. Subramanian, P. Karthikeyan, and N. Jagga Raju, "Modelling an electropneumatic brake system for commercial vehicles," IET Electrical Systems in Transportation, vol. 1, no. 1, pp. 41-48, 2011.

[2] G. Zhiwei, C. Cecati, and S. X. Ding, "A survey of fault diagnosis and fault-tolerant techniques part i: fault diagnosis with model-based and signal-based approaches," IEEE Transactions on Industrial Electronics, vol. 62, no. 6, pp. 3757-3767, 2015.

[3] A. Ilchmann, O. Sawodny, and S. Trenn, "Pneumatic cylinders: modelling and feedback force-control," International Journal of Control, vol. 79, no. 6, pp. 650-661, 2006.

[4] L. Wu and D. Ho, "Fuzzy filter design for ItÔ stochastic systems with application to sensor fault detection," IEEE Transactions on Fuzzy Systems, vol. 17, no. 1, pp. 233-242, 2009.

[5] G. H. B. Foo, X. Zhang, and D. M. Vilathgamuwa, "A sensor fault detection and isolation method in interior permanentmagnet synchronous motor drives based on an extended Kalman filter," IEEE Transactions on Industrial Electronics, vol. 60, no. 8, pp. 3485-3495, 2013.

[6] B. Pourbabaee, N. Meskin, and K. Khorasani, "Sensor fault detection, isolation, and identification using multiple-modelbased hybrid Kalman filter for gas turbine engines," IEEE Transactions on Control Systems Technology, vol. 24, no. 4, pp. 1184-1200, 2015.

[7] Z. Liu and H. He, "Model-based sensor fault diagnosis of a lithium-ion battery in electric vehicles," Energies, vol. 8, no. 7, pp. 6509-6527, 2015.

[8] P. Lu, L. Van Eykeren, E. J. Van Kampen, Q. P. Chu, and B. Yu, "Adaptive hybrid unscented Kalman filter for aircraft sensor fault detection, isolation and reconstruction," in AIAA guidance, navigation, and control conference, p. 1145, National Harbor, Maryland, 2014.

[9] C. Huang, G. Huang, W. Liu, R. Wang, and M. Xie, "A parallel joint optimized relay selection protocol for wake-up radio enabled WSNs," Physical Communication, vol. 47, no. 3, article en8076509, p. 10320, 2021.

[10] R. J. Patton, "Fault detection and diagnosis in aerospace systems using analytical redundancy," in IEE Colloquium on Condition Monitoring and Fault Tolerance, p. 1, London, UK, 1990.

[11] R. Wang, Y. Cheng, and M. Xu, "Analytical redundancy based fault diagnosis scheme for satellite attitude control systems," Journal of the Franklin Institute, vol. 352, no. 5, pp. 19061931, 2015.

[12] M. Taiebat and F. Sassani, "Distinguishing sensor faults from system faults by utilizing minimum sensor redundancy," Transactions of the Canadian Society for Mechanical Engineering, vol. 41, no. 3, pp. 469-487, 2017.

[13] D. Li, Y. Wang, J. Wang, C. Wang, and Y. Duan, "Recent advances in sensor fault diagnosis: a review," Sensors and Actuators A: Physical, vol. 309, article 111990, 2020.

[14] Y. L. Ou, "Fault diagnosis with fuzzy expert system," Applied Mechanics and Materials, vol. 48, 2011.

[15] M. Geetha and J. Jerome, "Fuzzy expert system based sensor and actuator fault diagnosis for continuous stirred tank 
reactor," in 2013 International Conference on Fuzzy Theory and Its Applications (iFUZZY), pp. 251-257, Taipei, Taiwan, 2013.

[16] K. Xiong, C. Chan, and H. Zhang, "Detection of satellite attitude sensor faults using the UKF," IEEE Transactions on Aerospace and Electronic Systems, vol. 43, no. 2, pp. 480-491, 2007.

[17] F. Auger, M. Hilairet, J. M. Guerrero, E. Monmasson, T. Orlowska-Kowalska, and S. Katsura, "Industrial applications of the Kalman filter: a review," IEEE Transactions on Industrial Electronics, vol. 60, no. 12, pp. 5458-5471, 2013.

[18] E. A. Wan and R. Van Der Merwe, "The unscented Kalman filter for nonlinear estimation," in Proceedings of the IEEE 2000 Adaptive Systems for Signal Processing, Communications, and Control Symposium (Cat. No.00EX373), pp. 153-158, Lake Louise, AB, Canada, 2000.

[19] R. Van Der Merwe and E. A. Wan, “The square-root unscented Kalman filter for state and parameter-estimation," in 2001 IEEE international conference on acoustics, speech, and signal processing, pp. 3461-3464, Salt Lake City, UT, USA, 2001.

[20] L. Zhentong and H. Hongwen, "Sensor fault detection and isolation for a lithium-ion battery pack in electric vehicles using adaptive extended Kalman filter," Applied Energy, vol. 185, no. 2, pp. 2033-2044, 2017.

[21] A. Mirzaee and K. Salahshoor, "Fault diagnosis and accommodation of nonlinear systems based on multiple-model adaptive unscented Kalman filter and switched MPC and h-infinity loop-shaping controller," Journal of Process Control, vol. 22, no. 3, pp. 626-634, 2012.

[22] F. Sun, X. Hu, Y. Zou, and S. Li, “Adaptive unscented Kalman filtering for state of charge estimation of a lithium-ion battery for electric vehicles," Energy, vol. 36, no. 5, pp. 3531-3540, 2011.

[23] T. Nguyen, J. Leavitt, F. Jabbari, and J. E. Bobrow, "Accurate sliding-mode control of pneumatic systems using low-cost solenoid valves," IEEE/ASME Transactions on Mechatronics, vol. 12, no. 2, pp. 216-219, 2007.

[24] F. Alonge, F. D’Ippolito, and F. M. Raimondi, "Least squares and genetic algorithms for parameter identification of induction motors," Control Engineering Practice, vol. 9, no. 6, pp. 647-657, 2001.

[25] K. Myers and B. Tapley, "Adaptive sequential estimation with unknown noise statistics," IEEE Transactions on Automatic Control, vol. 21, no. 4, pp. 520-523, 1976.

[26] S. Yong and C. Han, "Adaptive UKF method with applications to target tracking," Acta Automatica Sinica, vol. 37, no. 6, pp. 755-759, 2011.

[27] Y. Yang and W. Gao, "An optimal adaptive Kalman filter," Journal of Geodesy, vol. 80, no. 4, pp. 177-183, 2006.

[28] L. Kunpeng and Z. Qinghua, "An improved sequential probability ratio test method for residual test," Electronics Optics \& Control, vol. 16, no. 8, pp. 36-39, 2009.

[29] A. Wald and J. Wolfowitz, "Optimum character of the sequential probability ratio test," The Annals of Mathematical Statistics, vol. 19, no. 3, pp. 326-339, 1948.

[30] M. H. Carpenter, D. Gottlieb, S. Abarbanel, and W. S. Don, "The theoretical accuracy of Runge-Kutta time discretizations for the initial boundary value problem: a study of the boundary error," SIAM Journal on Scientific Computing, vol. 16, no. 6, pp. 1241-1252, 1995. 